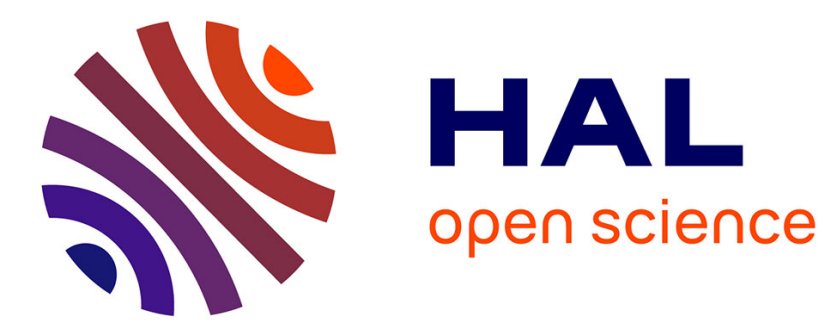

\title{
LES EMPLOIS DE L'ALUMINIUM EN LAITERIE
}

M. E. Drouilly

\section{To cite this version:}

M. E. Drouilly. LES EMPLOIS DE L'ALUMINIUM EN LAITERIE. Le Lait, 1921, 1 (5), pp.228-242. hal-00894671

\section{HAL Id: hal-00894671 \\ https://hal.science/hal-00894671}

Submitted on 1 Jan 1921

HAL is a multi-disciplinary open access archive for the deposit and dissemination of scientific research documents, whether they are published or not. The documents may come from teaching and research institutions in France or abroad, or from public or private research centers.
L'archive ouverte pluridisciplinaire HAL, est destinée au dépôt et à la diffusion de documents scientifiques de niveau recherche, publiés ou non, émanant des établissements d'enseignement et de recherche français ou étrangers, des laboratoires publics ou privés. 


\title{
LES EMPLOIS DE L'ALUMINIUM EN LAITERIE,
}

\author{
par M. E. DROUILLY, \\ Directeur du Département de l'Aluminium \\ a la Société des Tréfileries et Laminoirs du Havre.
}

L'aluminium est pour nous, à divers titres, un métal national ; d'abord parce que le premier lingot en fut obtenu au laboratoire par Sainte-Cliaire Deville, en 185̃ŏ, et que la fabrication industrielle en grand, par l'électrolyse, n'en commença, en 1888, que par l'application des procédés Héroult, autre savant français, mort en 1914; mais aussi, parce que les minerais de ce métal, alumines impures, connus sous le nom de bauxites rouges, sont particulièrement répandus sur notre sol de France, dans les départements du Var, des Bouches-duRhône, de l'Ariège, du Gard et de l'Hérault.

L'alumine propre à la fabrication de l'aluminium s'obtient en débarrassant chimiquement les bauxites rouges de l'oxyde de fer et des autres matières étrangères qu'elles contiennent, et, à son tour, l'aluminium se prépare en enlevant à l'alumine son oxygène par l'électrolyse ; cette dernière opération se fait dans les grandes installations hydro-électriques de notre massif montagneux alpin. C'est ainsi que l'aluminium est obtenu en France avec les produits du sol français, et sans le secours d'aucune contribution étrangère (1).

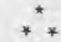

\section{Avantages physiques, mécaniques et chimiques de l'alu-} minium. - L'aluminium est susceptible de remplacer les autres

(1) Pour obtenir électriquement une tonne d'aluminium, il faut 2 tonnes d'alumine et 1 tonne de charboa (sous forme d'électrodes). Il faudrait en plus de 30 à 40 tonnes de charbon pour les chaudières, dans les pays ou la puissance électrique ne pourrait pas être demandée à la houille blanche.

Pour obtenir 2 tonues d'alumine, il faut 4 tonncs de bauxite et 8 tonnes de charbon. En raison de l'emplacement des mines de bauxite et a cause de l'intérêt qu'il y a à faire cette préparation chimique dans un endroit qui soit à la fois voisin de la mine et voisin de la mer, ces dernières 8 tonnes de charbon, pas plus que la tonne de charbon de cornues employée pour les électrodes, ne peuvent pas se remplacer par de la houille blanche.

Il faut donc effectivement brûler 9 tonnes de charbon pour obtenir une tonne d'aluminium, et ceci explique pourquoi l'aluminium en liugots qui avait atteint un prix èlevé en 1919 et en 1920 a vu son prix s'abaisser en même temps que celui du charbon, et est revenu â un taux qui permet de l'employer à la fabrication d'un grand nombre d'objets. 
métaux dans un grand nombre de cas, tant á eause de ses avantages physiques et mécaniques que de ses avantages chimiques.

Parmi ces avantages, citons, en premier lieu, sa légèreté; il est le plus léger des métaux usuels. En chiffres ronds, sa densité est 2,7, c'est dire qu'il est approximativement trois fois plus léger que le fer, plus de trois fois plus léger que le cuivre et l'étain. Sa couleur est d'un blane légèrement bleuté; il est susceptible de prendre un aspect mat sous l'influence d'un décapage, mais par le polissage, il prend un beau brillant.

Si l'aluminium pur est moins conducteur pour la chaleur que le cuivre, par contre, il est six fois plus conducteur que le fer.

Sa températuie de fusion est inférieure à celle de la fonte, du cuivre et du bronze, et il se prête facilement à la fusion des pièces moulées. Cependant comme sa résistance mécanique est sensiblement moindre à l'état fondu qu'à l'état laminé, on n'emploie guère les pièces fondues que pour les formes très compliquées, et qui comportent des bossages, des surépaisseurs en certains endroits etc..... ou pour les petites séries, tandis que pour toutes les pièces de forme régulière qui peuvent être obtenues par emboutissage mécanique ou hydraulique (pieces cylindriques, coniques, sphériques, par exemple), et toutes les fois qu'il s'agira de grandes séries, on préférera employer les tôles laminées, avec lesquelles on obtient la même résistance mécanique en utilisant une épaisseur deux à trois fois moindre et par conséquent un poids de métal deux à trois fois plus petit que si on devait faire les mêmes pièces en métal fondu.

L'aluminium est très ductile et malléable, et peut être amené sans se rompre à l'état de fils du diamètre d'un cheveu, et à l'état de feuilles laminées trois fois plus minces que le papier à cigarettes, ce qui l'a fait substituer à l'étain pour envelopper le ebocolat, le thé, les fruits confits (1), etc....

Il se soude facilement à lui-même, soit par la soudure autogène, qui est surtout employée pour les pièces fondues ou laminées, soit par la soudure électrique (qui est une soudure autogène sans apport de mélal extérieur) qui est surtout employée pour les fils comme pour la fabrication des maillons de chaines, par exemple. L'aluminium se file en tubes et en barres à la presse et à chaud; il se matrice à froid et à chaud; mais surtout il s'emboutit, s'étire, et se rétreint par des combinaisons appropriées de recuits (au besoin partiels) et d'opérations mécaniques ou hydrauliques, ce qui permet de le transformer économiquement en une foule d'objels dont les formes ne sont géné-

(1) Dans le conditionnement des poudres de lait, on prévoit qu'il puisse rendre de grands services. 
ralement réalisées avec les autres métaux qu'en ayant recours à l'agrafage, à la soudure, au ripetage, etc. . toutes opérations qui sont éminemment nuisibles lorsqu'on cherche à avoir des objets dont la surface doit être absolument lisse, ce que l'on recherche avec soin en laiterie. L'emboutissage, comme le laminage, augmente sa résistance mécanique, qui atteint facilement la moitié de celle de la tôle d'acier.

Parmi les avantages chimiques, disons que, pratiquement, il ne s'altère pas, sauf à l'état fondu, au contact de l'air et de l'eau. En réalité, il s'oxyde superficiellement, mais cette couche superficielle le préservant d'une oxydation ultérieure, les choses se passent comme s'il ne s'oxydait pas. Il est bien entendu que nous parlons ici du métal industriellement pur, connu sous le nom d'aluminium à $98 / 99 \%$, que l'on produit aujourd'hui couramment dans l'industrie, tandis que le métal allié avec des métaux capables de faire avec lui un couple voltaïque s'attaque assez facilement même à froid au contact de l'eau ou d'une atmosphère humide quand il n'est pas protégé par une couche isolante, par exemple, de la graisse, de l'huile, de la peinture. C'est ainsi qu'on a eu autrefois, dans la carrosserie automobile, des déboires, qu'on sait éviter aujourd'hui, d'abord en évitánt l'emploi de feuilles d'aluminium allié au cuivre, ensuite, en ne mettant pas des têtes de boulons, des vis ou des ferrures en contact direct avec l'aluminium. On n'a jamais eu ces inconvénients avec les carters d'automobiles qui bien qu'en aluminium allié, sont toujours recouverts d'huile ou de graisse, et on a su les éviter avec les capots, les ailes, les marchepieds, et toute la tôlerie en général, en ne se servant que d'aluminium à $98 / 99 \%$.

L'aluminium, contrairement à l'étain, résiste à l'acide sulfureux ; il résiste assez bien à l'acide sulfurique, tout à fait à l'acide nitrique pur (que l'on fabrique dans des appareils en aluminium); il résiste aux acides organiques, notamment á l'acide lactique, même après un contact prolongé.

L'aluminium n'a pas que de qualités, il a ses défauts. Il résiste peu au vinaigre, en présence du chlorure de sodium, à l'acide chl srhydrique et aux alcalis libres et carbonatés. Ces derniers peuvent néanmoins être employés pour le nettoyer, à condition d'être ensuite, soit neutralisés, soit complètement éliminés par des lavages à grande eau qui arrêtent l'attaque. On peut cependant prévoir qu'un jour, il pourra être utilisé, même pour le transport de ces corps, à conditiond'être protégé par certains enduits à base de laque de Chine ou de bakélite; on nous signale que des résultats très intéressants ont été obtenus par ces recouvrements pour l'emballage de la moutarde et de certains produits très salés. 
L'aluminium est susceptible de se cuivrer et de se nickeler par voie galvanique, et de recevoir des patines de diverses couleurs, soit par voie galvanique, soit par voie chimique, soit par une combinaison de ces deux procédés.

Après ce préambule nécessaire, voyons maintenant l'utilisation de l'aluminium en laiterie. La partie scientifique de ce sujet a fait l'objet d'une conférence récente ( 1 ) de M. Trillat, ehef de laboratoire à l'Institut Pasteur, qui a montré, comme étant le résultat d'un grand nombre d'expériences, que l'aluminium n'est attaqué ni par l'acide lactique, ni par aucan des produits que le lait contient à l'état naturel, ni par aucun de ceux auxquels le lait peut donner naissance après altération. M. Trillat a conclu en disant que l'aluminium n'a jamais donné de déboires qu'aux personnes qui ont voulu trop lui demander. N'en est-il pas de mème de toutes choses?

Le côté mécanique des emplois de l'aluminium en laiterie est de première importance. Quand on doit utiliser un récipient d'une certaine résistance mécanique, la première chose à faire est de calculer l'épaisseur qu'il faut donner à l'aluminium pour qu'il réponde à cette résistance. Encore faut-il ici marquer une différence bien nette entre les récipients qui ne sont destinés à servir qu'une seule fois, telles les boites utilisées pour le lait concentré, et ceux qui doivent faire un service journalier. Parmi ces derniers, nous devrons même distinguer, en laiterie, entre les ustensiles destinés aux transports, qui seront de ee fait l'objet de cahots, de heurts violents, de manipulations souvent brutales, et ceux qui, appartenant à la laiterie et n'en sortant pas, sont de ce fait moins exposés aux choes vigou. reux (2).

(1) Analysée dans " Le Lait ", n* 4, p. 214, 1921.

(2) En ce qui concerne les récipients qui étaient autrefois destinés à ne servir qu'une seule fois, il semble se produire depuis quelque temps une orientation nouvelle qu'il y a lieu d'encourager. Les boites en fer blanc étaient aussi minces que possible et leur procédé de fabrication par soudure ou agrafage avec joint étanche de caoutchouc non vulcanisè ne permettait pas, après vidange de leur contenu, de les transformer en articles de ménage. Pour la confiture, le miel et les conserves sucrées, on a déjà substitué l'aluminium au fer blane dans une large mesure, en employant des boites embouties en aluminium, fabriquées sans soudure et sans agrafage, et qui par conséquent sont étanches et vont sur le feu. Au lieu du procédé simple, mais rétrograde, qui consiste à souder le couvercle des boites en fer blanc après remplissage (et je ne parle pas ici des empoisonnements causés par les mauvaises soudures) et qui oblige, lorsqu'on ouvre ces boites à détruire le couvercle, voire même la boite, en blessant souvent l'opérateur qui n'a pas sous la main l'instrument approprié pour déchiręr le couvercle, on a employé des couvercles 
Le pot laitier. - Parmi les ustensiles de laiterie, le pot est celui qui est le plus employé. C'est son étude que nous allons aborder.

Laluminium, comme tous les métaux, rencontre devant lui, en laiterie, deux terribles adversaires :

a) La brutalité.

b) La malpropreté.

a) La brutalité est une habitude avec laquelle il faut absolument compter; elle est dans la nature humaine. Nousnous inclinons devant elle et nous ne parlerons donc que des pots qui peuvent être brutalisés. Souhaitons toutefois qu'ils le soient moins que les pots en fer, quoique nous ne l'espérions pas. Au surplus, des expériences des plus dures auxquelles nous avons soumis devant témoins des pots en aluminium nous donnent la plus grande confiance. Des pots de ø litres remplis d'eau ont été jetés sur un pavé de silex:

$\left.1^{\circ}\right)$ D'une hauteur de $2 \mathrm{~m} .50$ : cela ne leur a rien fait.

2.) D'une hauteur de $7 \mathrm{~m}$. 50 : légère bosselure.

$\left.3^{c}\right)$ D'une hauteur de 15 mètres : bosselure très marquée, mais le pot n'a pas été crevé.

Nous avons également vu donner de forts coups de talon sur le corps d'un pot de 20 litres, vide, sans réussir à lui faire plus que des enfoncements locaux d'un millimètre de profondeur; les clous du talon avaient laissé une marque très nette sur l'aluminium, témoignant par là de la violence du coup.

Nous souhaitons, avons-nous dit, que ces pots soient moins brutalisés que les pots en fer, parce que l'effort pour les soulever étant moindre, il est probable que les chocs le seront également. Enfin, ajouterons-nous, bien que l'aluminium n'ait pas besoin pour réussir d'un traitement privilégié de la part des garȩons laitiers, nous estimons que ceux-ei ne doivent pas être forcément plus brutaux que

à emboitement en aluminium, que l'on peut rendre complètement étanches avec des joints si c'est nécessaire; on réalise alors la fermeture avec des bagues serties, faciles à casser, si bien qu'à l'ouverture on ne détruit ni le couvercle ni la boite. Comme les fabricants de conserves peuvent acheter ces casseroles d'aluminium aux meilleures conditions possibles, en raison des quantités importantes qui leur sont nécessaires, et qu'ils ne prennent aucun bénéfice sur l'emballage, leurs clients ont ainsi le moyen de se procurer des ustensiles de ménage à bon marché. C'est ainsi qu'on nous a signalé que des boites à lait de 1 litre étaient rendues pleines de confitures chez l'épicier à un prix à peine supérieur au prix auquel elles sont vendues vides dans les bazars.

Nous nous attendons à voir bientôt vendre le beurre dans de semblables casseroles, l'huile d'olive dans des boites à lait de 5 litres et de 10 litres qui pourront être ensuite utilisées pour le lait.

Nous ne doutons pas également qu'il en pourra être de même pour les laits en poudre, les farines lactées, et même les laits concentrés pour lesquels la timbale d'aluminium semble offrir un mode d'emballage tout indiqué, 
les garçons épiciers, qui livrent le vin dans des bouteilles de verre. La brutalité de certaines personnes, en laiterie, n'est souvent qu'un masque pour leur cupidité. En même temps qu'un objet de transport, le pot laitier est une mesure, et si un pot en fer de 20 litres servant tous les jours au ramassage, arrive, sous l'influence de bosselures déterminées volontairement, nous sommes-nous laissé dire, à ne plus contenir que 19 litres $3 / 4$, cela fait 50 à 60 litres que ce pot rappurte par an à celui qui l'a bosselé. Ce sont des moeurs qu'il n'est pas indispensable de tolérer avec les pots en aluminium, car il suffit d'avoir une enclume ayant la forme extérieure du pot pour pouvoir remettre aisément ceux-ci en forme, en les frappant intérieurement avec un marteau de bois dur ou même avec un marteau planeur en acier. Ceci ne pourrait pas se faire avec un pot étamé sans enlever l'étain dont le fer est recouvert et sans provoquer ultérieurement et très vite la formation d'un dépót de rouille à cet endroit.

Avec l'aluminium, il n'y a pas de couche superficielle que l'on risque de détruire, et si on demande aux pots laitiers de ne pas pouvoir être bosselés par les chocs dus à une manutention ordinaire, on devra aussi avoir soin de les débosseler toutes les fois qu'une bosse aura pu se produire accidentellement ou volontairement.

Si l'accident est provoqué par une pointe et fait un trou, il est facile de réparer par un débosselage suivi d'un point de soudure autogène. Si l'accident compromet tout à fait la destination économique du pot, celui-ci aura encore une valeur pour la refonte, et les fabricants donnent un poids de poterie neuve en échange d'un poids quatre fois plus fort de pots usagés et incomplets qui leur sont rendus.

La brutalité toujours à envisager dans les manutentions a donc amené à étudier quelle était l'épaisseur qu'il fallait donner au pot laitier en aluminium. Des expériences ont été faites simultanément dans plusieurs pays et, pendant la guerre, c'est au Danemark, pays où les industriels n'avaient pas leur attention retenue par d'autres fabrications plus urgentes, qu'elles ont pu être poursuivies avec le plus de méthode.

Les premiers pots étaient en aluminium mince fretté par plusieurs cercles en acier ; ensuite, on a lait des pots à double paroi, la paroi intérieure étant en aluminium et la paroi extérieure en tole d'acier, mais très vite on en est venu à employer l'aluminium seul, en partant d'une disque en tôle de ce métal suffisamment épais pour aooir une résistance au moins égale à celle des pots en fer étamé dont onasait $\therefore$ 'habitude de se servir.

C'es derniers pots ont donné d'excellents résultats en s'arrêtant à une épaisseur de tôle de 3 millimètres ; c'est aussi cette épaisseur qui a été choisie par les fabricants français. Les fabricants danois pos- 
sèdent à l'heure actuelle des références sérieuses sur la façon dont leur poterie a résisté à l'usage journalier pendant quatre annẻes, mais chacun, aimant à avoir sur la matière une expérience personnelle, les fabricants français ont mis gratuitement à l'essai un certain nombre de pots dans diverses régions ; ils se mettent à la disposition de toutes les personnes que la question intéresse pour leur fournir même un seul pot, dont l'envoi peut facilemènt être fait par colis postal, puisqu'il ne pèse que $41 / 2$ kilog.

b) Contre la malpropreté, il n'y a pas non plus à espérer qu'un changement radical puisse être obtenu du jour au lendemain, bien que l'on puisse prévoir qu'avec le temps il sera possible de mieux s'éduquer soi-même, ce qui permettra ensuite de mieux éduquer le personnel.

Certains auteurs ont indiqué la nécessité (1) absolue qu'il y avait pour les personnes qui ramassent le lait à remettre elles-mêmes aux producteurs des pots parfaitement nettoyés et séchés. Il faut donc fabriquer des pots dont la surface intérieure soit parfaitement lisse, dont tous les angles soient arrondis, et dont les pentes soient calculées de façon à permettre l'écoulement des eaux de lavage en plaçant les pots retournés sur des claies d'égouttage.
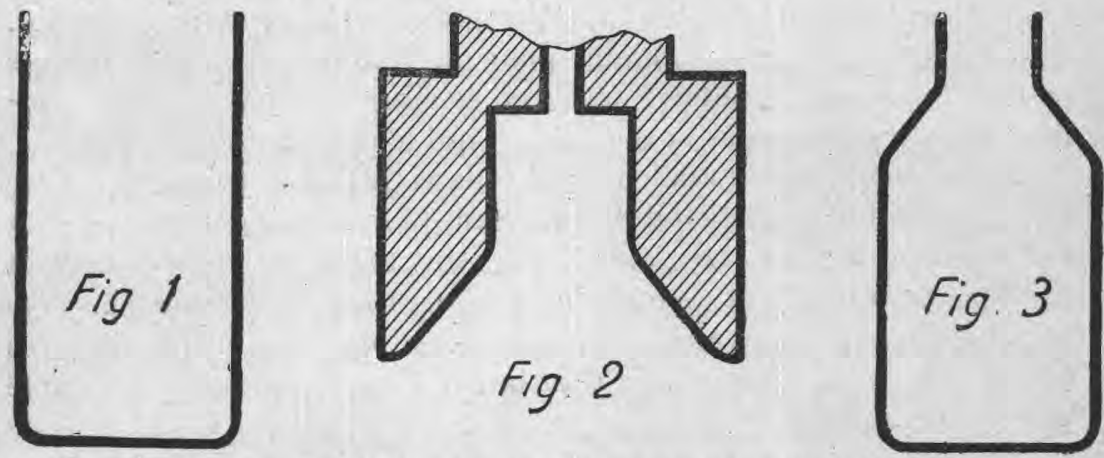

Ce sont là des conditions qu'il est difficile de réaliser complètement toutes les fois que l'on est obligé de faire appel au rivetage ou à la soudure. Or, l'aluminium est un métal qui se prête parfaitement à épouser les formes les plus diverses sans avoir recours à la soudure. Nous donnons la coupe des opérations successives par lesquelles passe la fabrication d'un pot laitier de 20 litres dans les usines du Hâvre.

Les feuilles laminées à $3 \mathrm{~m}$./m. d'épaisseur, sont découpées en

(1) Voir "Le Lait ", analyses diverses dans les no6 2, 3 et 4. 
disques du diamètre nécessaire, puis recuites ; elles sont alors embouties et étirées sur des presses puissantes qui leur lonnent la forme d'une marmite cylindrique de 26 centimètres de diamètre et de כ๊ centimètres de hauteur (figure 1). Les poinçons d'étirage étant légèrement coniques pour permettre de déchausser plus facilement la pièce qui vient d'être étirée, l'épaisseur de ce produit embouti est légèrement plus faible dans la partie ouverte que l'épaisseur du disque qui a servi de point de départ.

Par des forcements à la presse hydraulique de cette partie ouverte dans des matrices successives ayant la forme d'entonnoirs coniques terminés par des eylindres d'un diamètre de plus en plus faible (figure 2), on arrive, à la suite d'un certain nombre d'opérations, à donner au pot la forme de la figure 3 , le collet $\mathrm{C}$ étant parfaitement eylindrique. C'est iei que l'on voit d'une façon remarquable combien l'aluminium est un métal qui, peut-on dire, a bon caractère et combien les praticiens qui savent le manipuler peuvent le pétrir à leur gré. L'épaisseur initiale étant 3 millimètres, les bords du cylindre embouti n'avaient plus que 2,0 millimètres; l'épaisseur du collier, après les diverses opérations de retreint indiquées ci-dessus, est cependant redevenue à nouveau à 3 millimètres au minimum.

Il laut alors placer les frettes, qui sont également embouties dans des tòles de 3 millimètres d'épaisseur et qui peuvent être faites dans un alliage d'aluminium à la fois léger et très résistant, car comme elles n'entrent pas en contact avec le lait, il n'est pas indispensable qu'elles soient en aluminium pur.

La frette inférieure est obtenue par l'emboutissage d'un disque en forme de casserole (figure 4) et est transformée à la presse hydraulique selon la figure $\breve{.}$. Elle présente alors un disque intérieur qui dans les pots ordinaires est découpé et employé pour fabriquer le couvercle. En ne découpant pas ce disque, le frettage donne un double fond, et en donnant à cette frette une hauteur suffisante, elle permettrait même d'emprisonner entre ces deux fonds en aluminium, un troisième fond en bois contreplaqué, qui amortirait les choes et empècherait le bosselage et le perçage du fond dans les manulentions les plus brutales (figure 6).
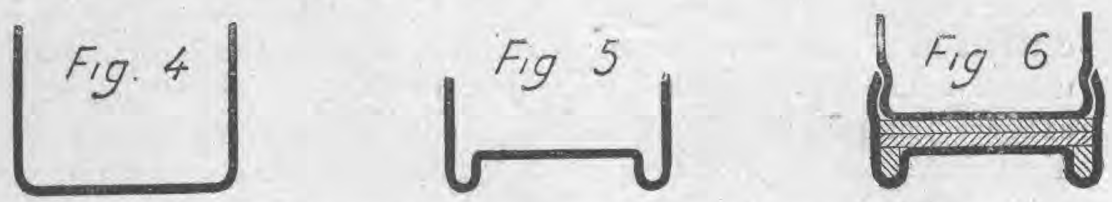

En général, le pot est suffisamment protégé par le bourrelet de la frette inférieure et le deuxième et le troisième fonds ne sont pas 
nécessaires. Au besoin, ce bourrelet peut être fourré au moyen d'une baguette en bois tourné ou en bois toupillé.

La frette est placée pendant quelques secondes dans un bain de plomb maintenu entre 400 et 420 degrés(température du bois fumant), elle reçoit ensuite la fourrure en bois du bourrelet, puis est placée sur le fond de la boîte. En la refroidissant brusquement avec de l'eau, on obtient un serrage énergique de la frette sur la boite qui suffit à assurer son adhérence sans le secours de la soudure, ni d'aucun rivet.

S’il fallait remplacer cette frette après un long usage, il suffirait de la couper au burin, puis de mettre à chaud une nouvelle frette en opérant exactement comme la première fois.

La frette du milieu, qui est découpée dans la même pièce emboutie que la frette inférieure, est rendue solidaire de la frette du collier par les anses. Les anses sont en tube d'aluminium (ou en alliage résistant et léger, genre duralumin). On les emplit de sable, de plomb ou de résine pour leur donner à chaud la forme convenable, puis on les vide et on aplatit leurs deux extrémités que l'on fixe fortement sur les deux frettes supérieures au moyen de deux rivets dont la tête fraisée affleure à l'intérieur des frettes.

La frette du collier a une hauteur égale à celle du collier lui-même, de façon à renforcer l'épaisseur de l'ouverture qui atteint ainsi un total de 6 millimètres. Elle porte en creux ou en relief les marques distinctives des laiteries, ce qui évite de créer pour ces marques dans le corps de la boite, une dépression toujours difficile à nettoyer parfaitement. Cette frette porte également une attache rivée pour la chaine du couvercle.

Le harnais composé des deux frettes supérieures, réunies par les anses de la chaine et du couvercle, est placé à chaud sur la boîte, puis refroidi comme il a été indiqné pour la frette intérieure.

Ensuite on opère le forcement de l'ouverture de la boite, en introduisant successivement dans cette ouverture, au moyen d'une presse hydranlique, deux cônes en acier de dimension appropriées, dont le premier, qui est le moins oblique, appuie fortement la paroi de la boite sur sa frette, et dont le second qui a exactement le même cône que le couvercle, n'appuie que sur 15 à 20 millimètres et limite à cette hauteur la surface de frottement de celui-ci. On obtient donc une ouverture qui affecte la forme de la figure 7 (ici les angles sont exagérés) et qui montre que la frette sur laquelle les anses sont fortement rivées fait partie intégrante de la boite, sans qu'il y ait soudure, et sans que les rivets apparaissent à l'intérieur (disposition brevetée). 
Le couvercle est un embouti conique à bord rabattu (figure 8) dans lequel on loge, avant de le rabattre, un cercle en fil d'aluminium de $14 \mathrm{~m}$./m. de diamètre Il est d'une très grande robustesse et on peut ainsi frapper en tous ses points avec un levier de fer pour obtenir, soit la fermeture, soit l'ouverture du pot. La barrette du couvercle est également retenue par ce fil à ses deux extrémités recourbées.

La chaîne est en fil d'aluminium et les maillons sont soudés électriquement.

Il existe une antre forme de couvercle, faite à la demande de certains fromagers qui ne désirent pas de chaine et veulent des couvereles interchangeables. Le meilleur moyen d'assurer cette interchangeabilité en économisant le temps, était de leur donner nettement deux millimètres de jeu et de réaliser l'herméticité du pot par un bracelet de caoutchouc qui doit être comme tout le reste, lavé soigneusement à chaque fois (figure 9).
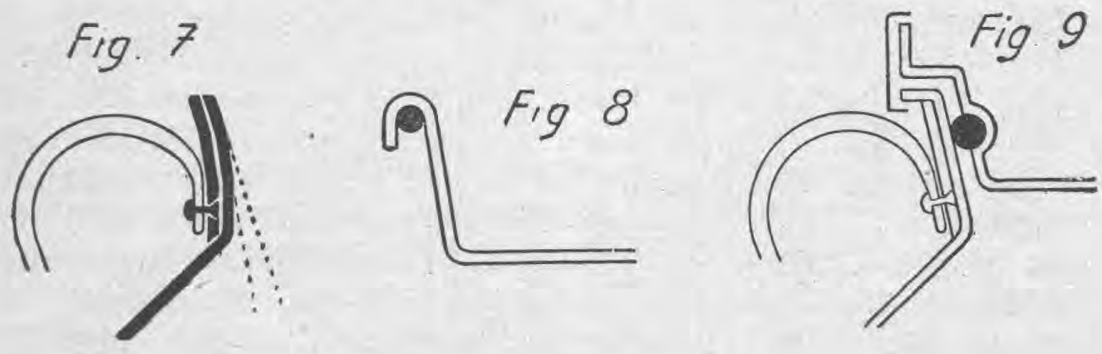

Pour fixer le couvercle à la boîte, on a fait venir un rebord extérieur sur le collier et le couvercle est maintenu en place par un collier amovible, à rigole et à charnière, qui emprisonne les bords de la boîte et du couvercle et qui est maintenu à sa position de fermeture par un système d'attache d'une grande simplicité.

En résumé, les caractéristiques du pot en aluminium sont celles-ci :

$1^{\circ}$ ) Ce pot (en tenant comple de son échange à quatre vieux pour un neut lorsqu'il est hors de service) est à peine plus cher à acheter qu'un pot en tôle étamée.

$2^{\circ}$ ) Il coûte beaucoup moins cher qu'un pot en fer étamé, si l'on ajoute au prix d'achat de celui-ci, le montant des frais de réétamage auxquels il entrainera pendant sa carrière.

$3^{\circ}$ ) Sa carrière est au moins égale à celle d'un pot en fer étamé.

$\left.4^{\circ}\right)$ Il n'est pas susceptible de rouiller.

$\left.5^{\circ}\right)$ Il pèse environ 4 kilos $1 / 2$ contre 7 kilos pour le pot en tôle étamée et l'économie de 2 kilos $1 / 2$, soit 5 kilos par voyage 'aller et retour, aura vite'remboursé son prix:d'achat. 
$\left.6^{\circ}\right)$ Comme il ne peut pas plus rouiller extérieurement qu'intérieurement, il n'y a aucun inconvénient à le mettre dans l'eau chaude ou froide.

$\left.7^{\circ}\right)$ Par les grandes chaleurs, il suffit de l'entourer d'un paillasson ou d'une enveloppe de feutre ou du jute qu'on arrose pour qu'il forme alcarazas ; grâce à la grande conduetibilité de l'aluminium, le lait est maintenu à une température assez basse, aussi bien pendant le transport que pendant les expositions au soleil dans les champs.

Pour tous ces avantages, nous souhaitons voir l'emploi du pot en aluminium se généraliser dans toutes les installations laitières.

La seule polémique digne d'attention qui se soit élevée est relative à la qualité de l'aluminium qui doit être employé pour le pot. L'avis de M. Trinlat est que l'on doit fixer son choix sur la qualité industrielle $98 / 99 \%$. En effet, on est certain d'en obtenir à volonté, tandis que la qualité extra-pure est plus chère, plus rare, et aussi moins résistante anx chocs.

La petite quantité de fer contenue dans cet aluminium industriel contribue, en effet, à en augmenter la résistance, et si elle apparaît sous forme d'un dépôt grisâtre à la suite d'un certain nombre de lavages à la soude, ce dépòt forme une patine quì ne se mélange pas au lait et qui n'a aucune influence sur sa qualité. Il est d'ailleurs facile de l'enlever pendant qu'il est encore humide, à la suite d'un lavage au carbonate de sodium, soit par un brossage énergique, soit par un lavage à l'acide sulfurique très éter du d'eau.

Sur une demande formelle et pour des quantités appréciables, il pourrait être fait des pots dont le vase intérieur serait en aluminium extra pur, qui resterait blane malgré les lavages alcalins, mais nous ne pensons pas que cela ajouterait quoi que ce soit à la qualité du pot, ni à celle du lait.

Le pot que nous avons décrit et dont la contenance est de 20 litres se fait aussi actuellement en $\check{5}$ litres et $\mathbf{1 5}$ litres, et il se fera d'ici peu en 10 litres, 25 litres et 30 litres.

Le pot de un et deux litres pour la distribution à domicile. Dans le $n^{\circ} 3$ de cette Revue, nous lisons ce qui suit à la page 130, dans l'analyse d'un article de The Milk Industry:

“ Les journaux politiques, entre autres The Times, agitent la a question de la pasteurisation obligatoire et de la vente en boua teilles également obligatoire. La vente en bouteilles ne peut pas " ètre rendue obligatoire, fait remarquer l'article d'une façon géné( rale, tant que lon ne sera pas certain de trouver un nombre de ( bouteilles suffisant..., ete, ».

Or, ceci ne serait pas un écueil en France, où la question a été 
étudiée et mise ả point par des industriels qui seraient capables de produire, à bref délai, plus de 100.000 bouteilles de un ou deux litres par semaine si elles leur étaient demandées.

En quoi faut-il faire les bouteilles?

Nous entendions dire, par un de nos grands spécialistes des maladies de l'enfance, qu'il valait mieux donner aux enfants des laits concentrés suerés, de bonne marque, étendus d'eau et bouillis, que des laits quelconques délivrés sans aucune garantie. Or, la supériorité des bonnes marques de lait concentré sur les laits quelconques vendus sans garantie, tient à ce qu'ils sont préparés avec soin et placés dans des boîtes inviolables. Pourquoi ne pas en faire autant pour le lait naturel, d'une façon courante?

On pouvait songer à l'aluminium pour faire les récipients, mais le prix de l'aluminium était considéré alors comme trop élevé ; or, il ne l'est plus aujourd'hui.

Les boîtes de fer blanc mince vont bien pour le lait concentré parce qu'elles ne servent qu'une fois. Mais avec le lait ordinaire, liquide, il faut pasteuriser, tyndalliser ou stériliser et, dans ces conditions, on ne peut songer au fer blanc.

De leur côté, les bouteilles en verre sont lourdes, se cassent et ne se prêtent pas commodément aux opérations de chauffage et de brusque refroidissement qu'exigent une fabrication bien conduite en laiterie.

Nous avons sous les yeux 20 boîtes tronc-coniques de un litre en aluminium qui pèsent ensemble 5 kg. 400 , soit $2 \mathrm{~kg}$. de moins que le pot de 20 litres en fer étamé. Leur emploi n'entrainerait done aucun supplément de transport. Leur manutention, en caisses, pourrait être rendue aussi rapide que celle des pots laitiers, et d'ailleurs, comme le lait mis dans ces boites serait comestible pendant plusieurs jours avec une pasteurisation bien faite, et même pendant plusieurs mois s'il était stérilisé, il ne serait plus besoin d'utiliser les transports par grande vitesse et l'on pourrait attendre, pour en faire I expédition, d'en avoir un wagon complet que l'on acheminerait sur les villes par petite vitesse.

C'est donc en fait une réduction des frais de transport par voie de fer qui pourrait être obtenue par l'adoption de ces boîtes qui permettraient, de plus, de faire l'approvisionnement des grandes villes au moyen de lait prélevé dans les eampagnes les plus reculées et les plus mal desservies au point de vue des transports.

La boite de un litre que nous avons sous les yeux est emboutie et rétreinte dans des disques d'aluminium découpés dans des tôles de $1 \mathrm{~m},, \breve{~ d}$ d'épaisseur. Elle est conique, à angles arrondis, et étant faite sans soudure ni rivet, sa surface intérieure est parfaitement lisse. 
Une large ouverture en permet le nettoyage facile. Elle porte une anse, supportée par deux rivets fixés à un collier par le même procédé breveté que le collier de la boîte de 20 litres. Le bord de la boîte est rabattu et permet l'application d'un couvercle à étage ayant assez de jeu pour être interchangeable, et rendu étanche par un joint plat de eaoutchouc (pur Para, sans soufre). La fermeture hermétique est obtenue par le sertissage d'une bague analogue aux bagues utilisées pour les bouchages métalliques des eaux minérales et qui s'enlève avec la plus grande facilité au moment de l'emploi.

Ces bouteilles vont sur le feu, aussi bien qu'au bain-marie, et permettent d'éviter tout transvasement depuis le filtrage du lait consécutif à la traite jusqu'au moment où il est apporté chaud sur la table du consommateur.

Les bouteilles sont fermées hermétiquement après remplissage et les opérations de pasteurisation, de tyndallisation ou de stérilisation, ont ainsi lieu en vase clos, en conservant tous les gàz du lait. Ces opérations peuvent se faire dans les plus petites installations comme dans les plus grandes, par un simple chauffage dans des bains-marie appropriés.

Sous l'influence de la dilatation du liquide et des gaz contenus à l'intérieur de la boîte, les deux fonds plans, qui résistent moins bien à la pression que la partie conique de la boite, s'incurvent vers l'extérieur et, la pression atmosphérique ne les ramenant pas à leur forme primitive, il se crée à l'intérieur de la boìte une dépression qui appuie fortement le couvercle; l'ouverture du pot est ainsi rendue impossible sans opérer une rentrée d'air. On emploie pour cela des joints de caoutchouc qui débordent l'extérieur de la boîte et du couvercle, tout au moins en un point, et en tirant sur cette partie débordante, on effectue à la fois la rentrée d'air et le décollage du joint qui permet une ouverture aisée du couvercle.

Il suffit de ramener les fonds à avoir la courbe contraire pour que la boîte, une fois nettoyée, soit prête pour une nouvelle opération, ce qui peut se faire automatiquement par un simple dispositif de la machine à sertir les bagues. Les pliages nayant jamais lieu exactement au même endroit,ce mouvement de soufflet des fonds peut avoir lieu des milliers de fois sans détériorer la boite ni son couvercle.

En employant l'aluminium à la confection des bagues de sertissage qui sont remplacées à chaque fois, et en rendant celles-ci au fournisseur qui les utilise en les refondant, le prix de revient de la fermeture des boites de un et deux litres est d'environ 10 centimes, y compris le joint d'étain ou de caoutchouc pur qui doit être remplacé chaque fois. Cette dépense est faible, en raison de la sécurité qu'elle donne, 
Nous avons également sous les yeux des boîtes d'un demi et d'un quart de gallon (2 lit. 500 et 1 lit. 250 ), qui sont fabriquées pour les besoins du marché anglais.

$$
\text { ** }
$$

Le biberon en aluminium. - Enfin, nous parlerons d'un biberon en aluminium, d'une contenance de 150 grammes; son nettoyage est rendu facile par le fait qu'il se sépare en deux parties, réunies par une bague sertie, l'herméticité étant obtenue par l'interposition entre les deux parties d'un joint en caoutchouc pur ou mieux en étain pur; son prix est modique (inférieur à 1 franc) et il peut être utilisé comme le pot de un litre décrit ci-dessus, non seulement pour les enfants, mais encore pour toute personne qui n'ayant besoin que de 150 grammes de lait à un moment donné, ne voudrait pas entamer une boîte de un litre.
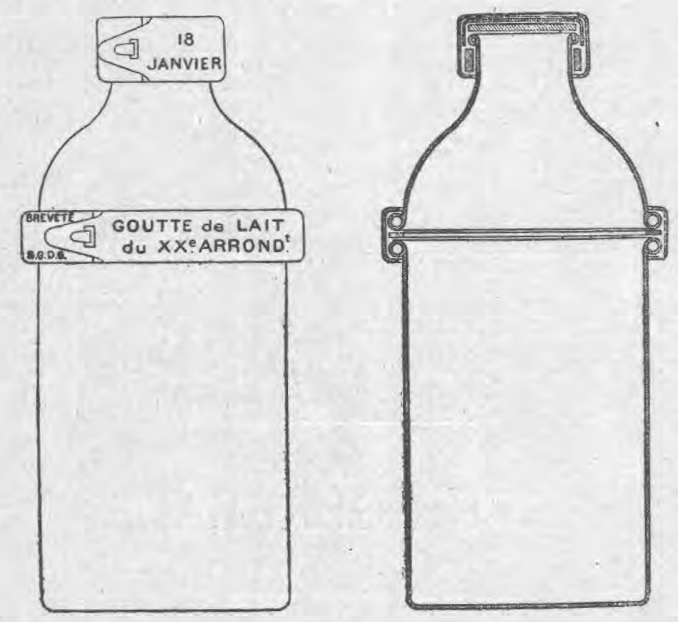

Si l'on pense que la tyndallisation et la stérilisation permettront de conserver pendant plusieurs mois le lait contenu dans ces petits pots, on pourra ainsi emporter en voyage un aliment que la simple inspection de la fermeture, rigoureusement hermétique, vous indiquera comme bon. On conseillera néanmoins aux mamans de goûter ce lait avant de le donner aux enfants, comme elles le font d'ailleurs avec des bouteilles en verre au travers desquelles on voit cependant le liquide.

$$
* *
$$

Nous nous sommes particulièrement étendu sur les divers pots, dont l'examen vient d'ètre fait, parce qu'étant des articles de grande 
consommation, ils peuvent être fabriqués en grande série et que l'industrie peut, par conséquent, les livrer aux conditions les meilleures; mais, en dehors des pots, nous voyons pour l'aluminium les emplois les plus larges en laiterie. Mesures, seaux, bacs, récipients pour maturation de la crème, etc., peuvent être faits en aluminium.

Le développement qu'a acquis son emploi dans les industries de fermentation et spécialement dans la brasserie, a amené à construire des laminoirs spéciaux pour le travail de ce métal et l'on trouve aujourd'hui des feuilles laminées dont les dimensions et les épaisseurs permettent, en ce qui concerne les bacs, de faire exécuter toutes les tailles voulues; la grande dim ension des tôles que l'on peut obtenir et la facilité de l'emboutissage permettront de réduire au minimum le nombre des soudures qui seront nécessaires pour l'établissement de ces bacs, soudures qui sont toujours coûteuses en raison du fait qu'il faut les lisser avec le plus grand soin.

Nous n'avons d'ailleurs pas épuisé la question et nous pensons y revenir lor'sque l'on aura industrialisé l'emploi des recouvrements de l'aluminium, en particulier par les bakélites et les laques de Chine qui semblent susceptibles de faire complètement corps avec les produits emboutis, au point de se plier avec eux lorsque ceux-ci reçoivent des chocs.

Mais en nous en tenant simplement pour le moment à ceux des articles pour lesquels l'emploi ne soulève aucune discussion, nous pensons avoir pour notre métal national une ère d'emplois nouveaux dont la laiterie pourra profiter dans la plus large mesure.

\section{REVUE GÉNÉRALE.}

\section{LA FERMENTATION LAGTIQUE,}

par E. KAYSER (Suite).

Influence de la température. - Il se manifeste ici une certaine spécificité, déterminée par la matière première qui subit la fermentation lactique.

Il existe une température minima, optima et maxima variable avec l'espèce ; ces températures sont comprises entre $10^{\circ}$ à $50^{\circ}$; certaines variétés du Str. cremoris poussent encore à $3^{\circ}$. 